\title{
Primal Hybrid Finite Element Methods for 2nd Order Elliptic Equations
}

\author{
By P. A. Raviart and J. M. Thomas
}

\begin{abstract}
The paper is devoted to the construction of finite element methods for 2nd order elliptic equations based on a primal hybrid variational principle. Optimal error bounds are proved. As a corollary, we obtain a general analysis of nonconforming finite element methods.
\end{abstract}

1. Introduction. Let $\Omega$ be a bounded open subset of $\mathbf{R}^{n}$, with a Lipschitz continuous boundary $\Gamma$. We consider the 2 nd order elliptic model problem

$$
\begin{aligned}
-\Delta u=f & \text { in } \Omega, \\
u=0 & \text { on } \Gamma,
\end{aligned}
$$

where $f$ is a given function of the space $L^{2}(\Omega)$. The usual variational form of problem (1.1) consists in finding $u \in H_{0}^{1}(\Omega)$ which minimizes the energy functional

$$
J(v)=\frac{1}{2} \int_{\Omega}|\operatorname{grad} v|^{2} d x-\int_{\Omega} f v d x
$$

over the space $H_{0}^{1}(\Omega)$.

Standard finite element methods for numerically solving problem (1.1) are based on this variational principle: they consist in first constructing a finite-dimensional subspace $V_{h}$ of the space $H_{0}^{1}(\Omega)$ made up with elementwise smooth functions which are continuous along the interelement boundaries and then in minimizing the energy functional $J(v)$ over the subspace $V_{h}$. Such conforming methods have been extensively studied and convergence results are now classical (see for instance Ciarlet [4], Strang and Fix [15]).

On the other hand, it has been noticed that one could weaken the requirement of interelement continuity for the functions of the space $V_{h}$ and still obtain a convergent finite element method. One gets the so-called nonconforming methods in which the space $V_{h}$ is no longer contained in $H_{0}^{1}(\Omega)$. For an analysis of some nonconforming methods for solving 2 nd order elliptic problems, we refer to Crouzeix and Raviart [6], Irons and Razzaque [8], Lesaint [9], Strang [14] .

In this paper, we shall use a more general approach in order to construct finite element approximations of problem (1.1). It is based on an extended variational principle, known as the primal hybrid principle, in which the constraint of interelement continuity has been removed at the expense of introducing a Lagrange multiplier. This type of method has been first introduced by the engineers (cf. Pian [10], [11],

Received December 26, 1975.

AMS (MOS) subject classifications (1970). Primary 65N15, 65N30.

Key words and phrases. Finite element methods, hybrid methods, Lagrange multipliers, nonconforming methods. 
Pian and Tong [12]) and can be viewed as a generalization of nonconforming methods.

Similarly, dual hybrid methods can be derived by using the complementary energy principle (cf. again [10], [11], [12]). For the numerical analysis of these methods, we refer to Thomas [16] , [17] , [18]. Hybrid methods for solving 4th order elliptic problems have been also analyzed: see Brezzi [1], [2] and Brezzi and Marini [3]. Finally, we refer to Fraeijs de Veubeke [7] for a general discussion of finite element methods including hybrid methods.

An outline of the paper is as follows. In Section 2, we describe the primal hybrid variational principle associated with problem (1.1); in Section 3, we define the method of approximation. Examples of triangular and quadrilateral hybrid elements are derived in Sections 4 and 5, respectively. The error analysis is given in Section 6 and, in Section 7, we discuss briefly the use of numerical integration in order to derive nonconforming methods.

Some results of this paper have been announced in [13]. For the sake of conciseness, we have omitted some proofs and developments; they will be found in [18].

Throughout this paper, we shall make a constant use of the Sobolev spaces

$$
H^{m}(\Omega)=\left\{v \in L^{2}(\Omega) ; \partial^{\alpha} v \in L^{2}(\Omega),|\alpha| \leqslant m\right\}
$$

provided with the norm and seminorms

$$
\|v\|_{m, \Omega}=\left(\sum_{|\alpha| \leqslant m} \int_{\Omega}\left|\partial^{\alpha} v\right|^{2} d x\right)^{1 / 2}, \quad|v|_{m, \Omega}=\left(\sum_{|\alpha|=m} \int_{\Omega}\left|\partial^{\alpha} v\right|^{2} d x\right)^{1 / 2} .
$$

Given a vector-valued function $\mathbf{q}=\left(q_{1}, \ldots, q_{n}\right) \in\left(H^{m}(\Omega)\right)^{n}$, we shall set:

$$
\|\mathbf{q}\|_{m, \Omega}=\left(\sum_{i=1}^{n}\left\|q_{i}\right\|_{m, \Omega}^{2}\right)^{1 / 2}, \quad|\mathbf{q}|_{m, \Omega}=\left(\sum_{i=1}^{n}\left|q_{i}\right|_{m, \Omega}^{2}\right)^{1 / 2}
$$

We shall denote by $H^{1 / 2}(\Gamma)$ the space of the traces $v_{\mid \Gamma}$ over $\Gamma$ of the functions $v \in$ $H^{1}(\Omega)$ and we shall define as usual

$$
H_{0}^{1}(\Omega)=\left\{v \in H^{1}(\Omega) ; v_{\mid \Gamma}=0\right\} .
$$

2. The Primal Hybrid Variational Formulation. Let $\bar{\Omega}=\bigcup_{r=1}^{R} \bar{\Omega}_{r}$ be a decomposition of the domain $\bar{\Omega}$ into subdomains $\bar{\Omega}_{r}$ such that:

(i) $\Omega_{r}$ is an open subset of $\Omega$ with a Lipschitz continuous boundary $\partial \Omega_{r}, 1 \leqslant r$ $\leqslant R$;

(ii) $\Omega_{r} \cap \Omega_{s}=\varnothing$ for $r \neq s$.

Clearly a function $v \in L^{2}(\Omega)$ belongs to the space $H_{0}^{1}(\Omega)$ iff

(a) the restriction $v_{r}$ of $v$ to the set $\Omega_{r}$ belongs to the space $H^{1}\left(\Omega_{r}\right)$,

(b) the traces of the functions $v_{r}$ and $v_{s}$ coincide on $\partial \Omega_{r} \cap \partial \Omega_{s}$;

(c) the trace of the function $v_{r}$ vanishes on $\partial \Omega_{r} \cap \Gamma, 1 \leqslant r \leqslant R$.

We want to relax the conditions (b) and (c). Hence, we introduce the space

$$
X=\left\{v \in L^{2}(\Omega) ; v_{r} \in H^{1}\left(\Omega_{r}\right), 1 \leqslant r \leqslant R\right\} \approx \prod_{r=1}^{R} H^{1}\left(\Omega_{r}\right)
$$

provided with the norm 


$$
\|v\|_{X}=\left(\sum_{r=1}^{R}\left\|v_{r}\right\|_{1, \Omega}^{2}\right)^{1 / 2}
$$

In order to characterize $H_{0}^{1}(\Omega)$ as a subspace of the space $X$, we first introduce the space

$$
\mathbf{H}(\operatorname{div} ; \Omega)=\left\{\mathbf{q} \in\left(L^{2}(\Omega)\right)^{n} ; \operatorname{div} \mathbf{q} \in L^{2}(\Omega)\right\}
$$

normed by

$$
\|q\|_{H(\operatorname{div} ; \Omega)}=\left(\|q\|_{0, \Omega}^{2}+\|\operatorname{div} q\|_{0, \Omega}^{2}\right)^{1 / 2}
$$

Given a vector-valued function $\mathbf{q} \in \mathbf{H}(\operatorname{div} ; \Omega)$, we may define its normal component $\mathrm{q} \cdot \nu \in H^{-1 / 2}(\Gamma)$ where $H^{-1 / 2}(\Gamma)$ is the dual space of $H^{1 / 2}(\Gamma)$ and $\nu$ is the unit outward normal along $\Gamma$. Moreover, we have Green's formula

$$
\forall v \in H^{1}(\Omega), \quad \int_{\Omega}\{\operatorname{grad} v \cdot \mathbf{q}+v \operatorname{div} \mathbf{q}\} d x=\int_{\Gamma} v \mathbf{q} \cdot v d \gamma
$$

where the integral $\int_{\Gamma}$ represents the duality between $H^{-1 / 2}(\Gamma)$ and $H^{1 / 2}(\Gamma)$.

Next, we define the space

$$
\begin{array}{r}
M=\left\{\mu \in \prod_{r=1}^{R} H^{-1 / 2}\left(\partial \Omega_{r}\right) ; \text { there exists a function } \mathbf{q} \in \mathbf{H}(\operatorname{div} ; \Omega)\right. \\
\text { such that } \left.\mathbf{q} \cdot \nu_{r}=\mu \text { on } \partial \Omega_{r}, 1 \leqslant r \leqslant R\right\},
\end{array}
$$

where $\nu_{r}$ is the unit outward normal along $\partial \Omega_{r}$. We may provide the space $M$ with the norm

$$
\|\mu\|_{M}=\underset{\mathrm{q} \in \mathrm{H}(\operatorname{div} ; \Omega) ; \mathrm{q} \cdot v_{r}=\mu \text { on } \partial \Omega_{r}, 1 \leqslant r \leqslant R}{ }\|\mathrm{q}\|_{H(\operatorname{div} ; \Omega)}
$$

Then we have

Lemma 1. A continuous linear functional $L$ on the space $X$ vanishes on $H_{0}^{1}(\Omega)$ iff there exists a unique element $\mu \in M$ such that

$$
\forall v \in X, \quad L(v)=\sum_{r=1}^{R} \int_{\partial \Omega_{r}} \mu v d \gamma
$$

Proof. By the Hahn-Banach theorem, any continuous linear functional on $H^{1}\left(\Omega_{r}\right)$ is of the form

$$
v \rightarrow \int_{\Omega_{r}}\left\{\sum_{i=1}^{n} q_{i} \frac{\partial v}{\partial x_{i}}+q_{0} v\right\} d x, \quad q_{i} \in L^{2}\left(\Omega_{r}\right), 0 \leqslant i \leqslant n .
$$

Hence, given a continuous linear functional $L$ on $X$, there exist $n+1$ functions $q_{i} \in$ $L^{2}(\Omega), 0 \leqslant i \leqslant n$, such that

$$
\forall v \in X, \quad L(v)=\sum_{r=1}^{R} \int_{\Omega_{r}}\left\{\sum_{i=1}^{n} q_{i} \frac{\partial v}{\partial x_{i}}+q_{0} v\right\} d x=0
$$


Assuming that $L$ vanishes on $H_{0}^{1}(\Omega)$, we get

$$
\forall v \in H_{0}^{1}(\Omega), \quad L(v)=\int_{\Omega}\left\{\sum_{i=1}^{n} q_{i} \frac{\partial v}{\partial x_{i}}+q_{0} v\right\} d x=0
$$

so that $q_{0}=\sum_{i=1}^{n} \partial q_{i} / \partial x_{i}$, in the sense of distributions in $\Omega$. Setting $q=\left(q_{1}, \ldots\right.$, $\left.q_{n}\right)$, we obtain $\operatorname{div} \mathbf{q}=q_{0} \in L^{2}(\Omega)$ so that $\mathbf{q} \in \mathbf{H}(\operatorname{div} ; \Omega)$ and

$$
\forall v \in X, \quad L(v)=\sum_{r=1}^{R} \int_{\Omega_{r}}\{\operatorname{grad} v \cdot \mathbf{q}+v \operatorname{div} \mathbf{q}\} d x
$$

Conversely, any linear functional of the form (2.9) is continuous on $X$ and vanishes on $H_{0}^{1}(\Omega)$.

Now, using the Green's formula (2.5) in each $\Omega_{r}$, we get from (2.9)

$$
\forall v \in X, \quad L(v)=\sum_{r=1}^{R} \int_{\partial \Omega_{r}} v \mathrm{q} \cdot \nu_{r} d \gamma
$$

Clearly, in (2.10), the function $q$ is not uniquely determined but the corresponding element $\mu \in M$ is unique. In fact, assume that

$$
\forall v \in X, \quad \sum_{r=1}^{R} \int_{\partial \Omega_{r}} \mu v d \gamma=0
$$

Then, we get, for all $r=1, \ldots, R$,

$$
\forall v \in H^{1}\left(\Omega_{r}\right), \quad \int_{\partial \Omega_{r}} \mu v d \gamma=0,
$$

which implies $\mu=0$ on $\partial \Omega_{r}$ by the surjectivity of the trace operator $v \rightarrow v_{\mid \partial \Omega_{r}}$ from $H^{1}\left(\Omega_{r}\right)$ onto $H^{1 / 2}\left(\partial \Omega_{r}\right)$. The proof of the lemma is now complete.

Consider the continuous bilinear form on $X \times M$

$$
b(v, \mu)=-\sum_{r=1}^{R} \int_{\partial \Omega_{r}}^{\mu v d \gamma}
$$

Then, as a consequence of Lemma 1 , we get the following characterization of the space $H_{0}^{1}(\Omega)$

$$
H_{0}^{1}(\Omega)=\{v \in X ; \forall \mu \in M, b(v, \mu)=0\} .
$$

We are now able to introduce the primal hybrid formulation of problem (1.1). Define the continuous bilinear form on $X \times X$

$$
a(u, v)=\sum_{r=1}^{R} \int_{\Omega_{r}} \operatorname{grad} u \cdot \operatorname{grad} v d x .
$$

We want to find a pair $(u, \lambda) \in X \times M$ such that

$$
\forall v \in X, a(u, v)+b(v, \lambda)=\int_{\Omega} f v d x
$$

$$
\forall \mu \in M, \quad b(u, \mu)=0
$$


Theorem 1. Problem (2.14), (2.15) has a unique solution $(u, \lambda) \in X \times M$. Moreover $u \in H_{0}^{1}(\Omega)$ is the solution of problem (1.1) and we have

$$
\lambda=\partial u / \partial \nu_{r} \text { on } \partial \Omega_{r}, 1 \leqslant r \leqslant R .
$$

Proof. Let $(u, \lambda) \in X \times M$ be a solution of (2.14), (2.15). Then, by (2.12) we have $u \in H_{0}^{1}(\Omega)$. Choosing $v \in H_{0}^{1}(\Omega)$ in (2.14) gives

$$
\forall v \in H_{0}^{1}(\Omega), \quad \int_{\Omega} \operatorname{grad} u \cdot \operatorname{grad} v d x=\int f v d x
$$

so that $u$ is the solution of problem (1.1). Conversely, let $u \in H_{0}^{1}(\Omega)$ be the solution of (1.1) and consider the continuous linear functional on $X$

$$
v \rightarrow \int_{\Omega} f v d x-a(u, v)
$$

it vanishes on $H_{0}^{1}(\Omega)$ so that, by Lemma 1 , there exists a unique $\lambda \in M$ such that

$$
\forall v \in X, \quad b(v, \lambda)=\int_{\Omega} f v d x-a(u, v) .
$$

Hence, the pair $(u, \lambda)$ is the solution of $(2.14),(2.15)$.

Now, since $f=-\Delta u$, we obtain by using the Green's formula (2.5) in each $\Omega_{r}$ with $\mathbf{q}=\operatorname{grad} u$

$$
\forall v \in X, \quad b(v, \lambda)=-\int_{\Omega} \Delta u v d x-a(u, v)=-\sum_{r=1}^{R} \int_{\partial \Omega_{r}} \frac{\partial u}{\partial \nu_{r}} v d \gamma
$$

so that $(2.16)$ holds.

Remark 1. Define the continuous quadratic functional on $X \times M$

$$
L(v, \mu)=J(v)+b(v, \mu) .
$$

Then, one can easily check that the solution $(u, \lambda) \in X \times M$ of problem (2.14), (2.15) may be characterized as the unique saddle-point of the functional $L(v, \mu)$ over $X \times M$, i.e.,

$$
L(u, \lambda)=\operatorname{Min}_{v \in X} \operatorname{Max}_{\mu \in M} L(v, \mu)=\operatorname{Max}_{\mu \in M} \operatorname{Min}_{v \in X} L(v, \mu) .
$$

Hence, $\lambda$ is the Lagrange multiplier associated with the constraint $u \in H_{0}^{1}(\Omega)$.

3. A Hybrid Finite Element Method. Let us now introduce a method of approximation of problem (1.1) based on the primal hybrid variational formulation (2.14), (2.15). Given two finite-dimensional spaces $X_{h}$ and $M_{h}$ which satisfy the inclusions

$$
X_{h} \subset X, \quad M_{h} \subset M,
$$

we define problem $\left(\mathrm{Q}_{h}\right)$ : Find a pair $\left(u_{h}, \lambda_{h}\right) \in X_{h} \times M_{h}$ such that

$$
\begin{gathered}
\forall v_{h} \in X_{h}, \quad a\left(u_{h}, v_{h}\right)+b\left(v_{h}, \lambda_{h}\right)=\int_{\Omega} f v_{h} d x, \\
\forall \mu_{h} \in M_{h}, \quad b\left(u_{h}, \mu_{h}\right)=0 .
\end{gathered}
$$

Before solving problem $\left(Q_{h}\right)$, we introduce the space 


$$
V_{h}=\left\{v_{h} \in X_{h} ; \forall \mu_{h} \in M_{h}, b\left(v_{h}, \mu_{h}\right)=0\right\} .
$$

Comparing with (2.12), the space $V_{h}$ appears to be an approximation of the space $H_{0}^{1}(\Omega)$. Notice however that $V_{h}$ is not in general a subspace of $H_{0}^{1}(\Omega)$. Then, we define problem $\left(\mathrm{P}_{h}\right)$ : Find $u_{h} \in V_{h}$ such that

$$
\forall v_{h} \in V_{h}, \quad a\left(u_{h}, v_{h}\right)=\int_{\Omega} f v_{h} d x .
$$

Since in general $V_{h} \not \subset H_{0}^{1}(\Omega)$, problem $\left(\mathrm{P}_{h}\right)$ is a nonconforming method for numerically solving problem (1.1).

Note that if $\left(u_{h}, \lambda_{h}\right) \in X_{h} \times M_{h}$ is a solution of problem $\left(Q_{h}\right), u_{h} \in V_{h}$ is a solution of problem $\left(\mathrm{P}_{h}\right)$. Moreover, we have the following result.

THEOREM 2. Assume that

$$
v_{h} \rightarrow\left\|v_{h}\right\|_{h}=a\left(v_{h}, v_{h}\right)^{1 / 2} \text { is a norm over } V_{h} .
$$

Then:

(i) problem $\left(\mathrm{P}_{h}\right)$ has a unique solution $u_{h} \in V_{h}$;

(ii) problem $\left(\mathrm{Q}_{h}\right)$ has a unique solution $\left(u_{h}, \lambda_{h}\right) \in X_{h} \times M_{h}$ iff the following compatibility condition holds

$$
\left\{\mu_{h} \in M_{h} ; \forall v_{h} \in X_{h}, b\left(v_{h}, \mu_{h}\right)=0\right\}=\{0\} .
$$

Proof. The existence and uniqueness of the solution $u_{h} \in V_{h}$ of problem $\left(\mathrm{P}_{h}\right)$ follows from the assumption (3.6) and the Lax-Milgram lemma. On the other hand, since problem $\left(\mathrm{Q}_{h}\right)$ is equivalent to a $N \times N$ linear system with $N=\operatorname{dim} X_{h}+\operatorname{dim} M_{h}$, the existence of the solution of problem $\left(Q_{h}\right)$ follows from the uniqueness. Thus, assume $f=0$. Necessarily $u_{h}=0$ so that $\lambda_{h}$ is characterized by the condition

$$
\forall v_{h} \in X_{h}, \quad b\left(v_{h}, \lambda_{h}\right)=0 .
$$

Therefore $\lambda_{h}=0$ iff condition (3.7) holds. $\square$

In the sequel, we shall assume that $\Omega$ is a bounded and polyhedral subset of $\mathbf{R}^{n}$. Let $T_{h}$ be a triangulation of the set $\bar{\Omega}$ with polyhedra $K$ whose diameters are $\leqslant h$. For any $K \in T_{h}$, we denote by $\partial K$ the boundary of $K$ and $\nu_{K}$ the unit outward normal along $\partial K$. We now use the decomposition

$$
\bar{\Omega}=\bigcup_{K \in T_{h}} K
$$

of the domain $\bar{\Omega}$ for defining a hybrid finite element method. The first step consists in constructng a finite-dimensional subspace $X_{h}$ of the space

$$
X=X\left(T_{h}\right)=\left\{v \in L^{2}(\Omega) ; \forall K \in T_{h}, v_{\mid K} \in H^{1}(K)\right\} .
$$

Let $\hat{K}$ be a reference polyhedron (for instance the unit right $n$-simplex or the unit hypercube). We assume for simplicity that each polyhedron $K \in T_{h}$ is the image of $\hat{K}$ through an affine invertible mapping $F_{K}$. We now introduce a finite-dimensional subspace $\hat{P}$ of the space $H^{1}(\hat{K})$ which satisfies the inclusion

$$
P_{k} \subset \hat{P} \text { for some integer } k \geqslant 1,
$$


where $P_{k}$ denotes the space of all polynomials of degree $\leqslant k$ in the $n$ variables $x_{1}$, $\ldots, x_{n}$.

Moreover, let us denote by $\hat{S}_{k}$ the space of all functions defined over $\partial \hat{K}$ whose restrictions to any $(n-1)$-dimensional face $\hat{K}^{\prime}$ of $\partial \hat{K}$ are polynomials of degree $\leqslant k$. Let us denote by $\hat{T}_{k}$ the space of all functions of $S_{k}$ which are continuous over $\partial \hat{K}$. We assume, in addition, that the space $\hat{P}_{\mid \partial \hat{K}}$ of the restrictions to the set $\partial \hat{K}$ of all functions of $\hat{P}$ satisfies the inclusion

$$
\hat{T}_{k} \subset \hat{P}_{\mid \partial \hat{K}}
$$

Then, we define

$$
P_{K}=\left\{v \in H^{1}(K) ; v=\hat{v}_{0} F_{K}^{-1}, \hat{v} \in \hat{P}\right\}
$$

and

$$
X_{h}=\left\{v \in L^{2}(\Omega) ; \forall K \in T_{h}, v_{\mid K} \in P_{K}\right\} .
$$

Note that the functions of $X_{h}$ do not satisfy any continuity constraint at the interelement boundaries.

The second step consists in constructing a finite-dimensional subspace $M_{h}$ of the space

$$
\begin{array}{r}
M=M\left(T_{h}\right)=\left\{\mu \in \prod_{K \in T_{h}} H^{-1 / 2}(\partial K) ; \text { there exists a function } \mathbf{q} \in \mathbf{H}(\operatorname{div} ; K)\right. \\
\text { such that } \left.\mathbf{q} \cdot \nu_{K}=\mu \text { on } \partial K, K \in T_{h}\right\} .
\end{array}
$$

We introduce a finite-dimensional subspace $\hat{S}$ of the space $L^{2}(\partial \hat{K})$ which satisfies the following properties:

$$
\begin{aligned}
& \hat{S}_{m} \subset \hat{S} \quad \text { for some integer } m \geqslant 0, \\
& \forall \hat{\alpha} \in \hat{S}_{0}, \quad \hat{\alpha} \hat{S}=\{\hat{\alpha} \hat{\mu} ; \hat{\mu} \in \hat{S}\} \subset \hat{S} .
\end{aligned}
$$

Now, for any $K \in T_{h}$, we set

$$
S_{\partial K}=\left\{\mu \in L^{2}(\partial K) ; \mu=\hat{\mu}_{0} F_{K}^{-1}, \hat{\mu} \in \hat{S}\right\} .
$$

Then, we define

$$
\begin{aligned}
M_{h}=\left\{\mu \in \prod_{K \in T_{h}} S_{\partial K} ; \mu_{\mid \partial K_{1}}+\mu_{\mid \partial K_{2}}=0 \text { on } K_{1} \cap K_{2}\right. \text { for } \\
\text { every pair of adjacent elements } \left.K_{1}, K_{2} \in T_{h}\right\} .
\end{aligned}
$$

One easily checks that $M_{h}$ is indeed a subspace of $M$.

Next, we want to give some simple sufficient conditions for hypotheses (3.6) and (3.7) to hold. We begin with

Lemma 2. Assume that the inclusion (3.12) holds. Then, condition (3.6) is satisfied.

Proof. Let $v_{h}$ be a function of $V_{h}$ such that 


$$
\left\|v_{h}\right\|_{h}^{2}=a\left(v_{h}, v_{h}\right)=\sum_{K \in T_{h}} \int_{K}\left|\operatorname{grad} v_{h}\right|^{2} d x=0 .
$$

Clearly, the function $v_{h}$ is constant in each element $K \in T_{h}$. Now, let $K^{\prime}$ be a $(n-1)$ dimensional common face of two adjacent elements $K_{1}, K_{2} \in T_{h}$. Define $\mu_{h} \in$ $\Pi_{K \in T_{h}} L^{2}(\partial K)$ by

$$
\begin{gathered}
\mu_{h \mid \partial K_{1}}=\left\{\begin{array}{ll}
1 & \text { on } K^{\prime}, \\
0 & \text { on } \partial K_{1} \backslash K^{\prime},
\end{array} \quad \mu_{h \mid \partial K_{2}}= \begin{cases}-1 & \text { on } K^{\prime}, \\
0 & \text { on } \partial K_{2} \backslash K^{\prime},\end{cases} \right. \\
\mu_{h \mid \partial K}=0 \quad \text { for } K \neq K_{1}, K_{2} .
\end{gathered}
$$

Since $\hat{S}_{0} \subset \hat{S}$, the function $\mu_{h}$ belongs to the space $M_{h}$. Hence, denoting by $c_{i}$ the constant value of $v_{h}$ in $K_{i}, i=1,2$, and using the definition (3.4) of the space $V_{h}$, we get

$$
0=b\left(v_{h}, \mu_{h}\right)=\left(c_{1}-c_{2}\right) \int_{K^{\prime}} d \gamma
$$

so that $c_{1}=c_{2}$. Therefore, the function $v_{h}$ is constant in $\Omega$.

Finally, let $K^{\prime}$ be a $(n-1)$-dimensional face contained in $\Gamma$. Define the function $\mu_{h} \in M_{h}$ by

$$
\mu_{h \mid K^{\prime}}=1, \quad \mu_{h}=0 \text { elsewhere. }
$$

We obtain

$$
0=b\left(v_{h}, \mu_{h}\right)=\int_{K}, v_{h} d \gamma
$$

so that $v_{h}=0$ in $\Omega$. This proves that $v_{h} \rightarrow\left\|v_{h}\right\|_{h}$ is a norm over the space $V_{h}$.

Since condition (3.6) is always satisfied, problem $\left(\mathrm{P}_{h}\right)$ has a unique solution $u_{h} \in V_{h}$. On the contrary, condition (3.7) which ensures the existence and uniqueness of the Lagrange multiplier $\lambda_{h} \in M_{h}$ is satisfied only for compatible choices of the spaces $\hat{P}$ and $\hat{S}$. In this respect, we have

Lemma 3. Assume that

$$
\left\{\mu \in \hat{S} ; \forall v \in \hat{P}, \int_{\partial \hat{K}} \mu v d \gamma=0\right\}=\{0\} .
$$

Then, condition (3.7) holds.

Proof. Using (3.16) and the definitions (3.10) and (3.14) of the spaces $P_{K}$ and $S_{\partial K}$ respectively, we get for all $K \in T_{h}$

$$
\left\{\mu \in S_{\partial K} ; \forall v \in P_{K}, \int_{\partial K} \mu v d \gamma=0\right\}=\{0\} .
$$

This in turn implies (3.7).

Remark 2. In fact, one can prove that, at least for some particular triangulation $T_{h}$ of the domain $\bar{\Omega}$, condition (3.16) is as well necessary for hypothesis (3.7) to hold. $\square$ 
The next two sections of this paper will now be devoted to the explicit construction of triangular and quadrilateral finite elements in $R^{2}$ which satisfy the compatability condition (3.16).

4. Examples of Hybrid Triangular Elements. Assume now $n=2$. We first consider the case of triangular elements: here $\hat{K}$ is a triangle with vertices $a_{i}, 1 \leqslant i$ $\leqslant 3$; we denote by $\lambda_{i}=\lambda_{i}(x), 1 \leqslant i \leqslant 3$, the barycentric coordinates of a point $x \in$ $\mathbf{R}^{2}$ with respect to the vertices $a_{i}$ of $\hat{K}$.

Lemma 4. Assume that $\hat{K}$ is a triangle. Let $k \geqslant 1$ and $m \geqslant 0$ be two integers. Then conditions

$$
\left\{\begin{array}{l}
\mu \in \hat{S}_{m} \\
\forall v \in \hat{T}_{k}, \int_{\partial \hat{K}} \mu v d \gamma=0
\end{array}\right.
$$

imply $\mu=0$ iff

$$
k \geqslant \begin{cases}m+1 & \text { when } m \text { is even, } \\ m+2 & \text { when } m \text { is odd. }\end{cases}
$$

Proof. Since $\hat{K}$ is a triangle, we have $\hat{T}_{k}=P_{k \mid \partial \hat{K}}$ and $\operatorname{dim}\left(\hat{S}_{m}\right)=3(m+1)$, $\operatorname{dim}\left(\hat{T}_{k}\right)=3 k$.

For $k \leqslant m$, we get $\operatorname{dim}\left(\hat{S}_{m}\right)>\operatorname{dim}\left(\hat{T}_{k}\right)$ so that conditions (4.1) cannot imply $\mu=0$.

Thus, let us assume $k \geqslant m+1$ and let $\mu$ satisfy conditions (4.1). Then, for all $v \in \hat{T}_{k}$, we get $\mu v \in \hat{S}_{2 k-1}$ so that the integral $\int_{\partial \hat{K}} \mu \nu d \gamma$ can be computed exactly in terms of the values of the function $\mu v$ at the $(k+1)$ Gauss-Lobatto quadrature points of each side of $\partial \hat{K}$. Denote by $\left\{a_{1}, a_{4}, \ldots, a_{k+2}, a_{2}\right\}$ (resp. $\left\{a_{2}, a_{k+3}, \ldots\right.$, $\left.a_{2 k+1}, a_{3}\right\},\left\{a_{3}, a_{2 k+2}, \ldots, a_{3 k}, a_{1}\right\}$ the set of $(k+1)$ Gauss-Lobatto points of the side $\left[a_{1}, a_{2}\right]$ (resp. $\left[a_{2}, a_{3}\right],\left[a_{3}, a_{1}\right]$ ). Clearly, for each $i=1, \ldots, 3 k$, there exists a unique function $v_{i} \in \hat{T}_{k}$ such that

$$
v_{i}\left(a_{j}\right)=\delta_{i j}, \quad 1 \leqslant j \leqslant 3 k
$$

Since conditions (4.1) are invariant by an affine invertible mapping, we may assume that the triangle $\hat{K}$ is equilateral. Then, replacing $v$ by $v_{i}, 1 \leqslant i \leqslant 3 k$, in (4.1) gives

$$
\mu\left(a_{i}\right), \quad 4 \leqslant i \leqslant 3 k
$$

and

$$
\left\{\begin{array}{l}
\mu_{12}\left(a_{1}\right)+\mu_{13}\left(a_{1}\right)=0 \\
\mu_{23}\left(a_{2}\right)+\mu_{21}\left(a_{2}\right)=0 \\
\mu_{31}\left(a_{3}\right)+\mu_{32}\left(a_{2}\right)=0
\end{array}\right.
$$

where $\mu_{i j}=\mu_{j i}$ is the restriction of $\mu$ to the side $\left[a_{i}, a_{j}\right]$.

For $k \geqslant m+2$, conditions (4.3) imply $\mu=0$. Hence, it remains only to consider the case $k=m+1$. Let 


$$
0=\xi_{0}<\xi_{1}<\cdots<\xi_{k-1}<\xi_{k}=1
$$

be the $(k+1)$ Gauss-Lobatto abscissae for $[0,1]$; we introduce the homogeneous polynomial of degree $k-1$ in the variables $\xi$ and $\eta$

$$
p_{k-1}(\xi, \eta)=\prod_{i=1}^{k-1}\left(\eta_{i} \xi-\xi_{i} \eta\right),
$$

where $\eta_{i}=1-\xi_{i}, 1 \leqslant i \leqslant k-1$. Since $\eta_{i}=\xi_{k-i}, 1 \leqslant i \leqslant k-1$, we get

$$
p_{k-1}(\xi, \eta)=(-1)^{k-1} p_{k-1}(\eta, \xi) \text {. }
$$

Thus, for $k=m+1$, conditions (4.3) exactly mean

$$
\mu_{i j}=c_{i j} p_{k-1}\left(\lambda_{i}, \lambda_{j}\right) \text { on }\left[a_{i}, a_{j}\right], \quad c_{i j}=(-1)^{k-1} c_{j i} .
$$

Using (4.6), conditions (4.4) become

$$
\left\{\begin{array}{l}
c_{12}+(-1)^{k-1} c_{31}=0 \\
c_{23}+(-1)^{k-1} c_{12}=0 \\
c_{31}+(-1)^{k-1} c_{23}=0 .
\end{array}\right.
$$

When $m=k-1$ is even, the linear system (4.7) has the unique solution $c_{12}=c_{23}=$ $c_{31}=0$, so that $\mu=0$. When $m=k-1$ is odd, (4.7) has nontrivial solutions so that conditions (4.1) do not imply $\mu=0$.

Remark 3. When $m=k-1$ is odd, we have proved that the space of functions $\mu$ which satisfy conditions (4.1) is one-dimensional. When $\hat{K}$ is equilateral, these functions $\mu$ are the functions of the form

$$
\left\{\begin{array}{l}
\mu_{12}=c p_{k-1}\left(\lambda_{1}, \lambda_{2}\right), \\
\mu_{23}=c p_{k-1}\left(\lambda_{2}, \lambda_{3}\right), \quad c \in \mathbf{R} . \\
\mu_{31}=c p_{k-1}\left(\lambda_{3}, \lambda_{1}\right),
\end{array}\right.
$$

We are now able to introduce

Example 1 . Let $k \geqslant 1$ be an odd integer. Given a reference triangle $\hat{K}$, we choose:

$$
\hat{P}=P_{k}, \quad \hat{S}=\hat{S}_{k-1} \quad(m=k-1) .
$$

Then, by Lemmas 2,3 and 4 , the corresponding problem $\left(Q_{h}\right)$ has a unique solution $\left(u_{h}, \lambda_{h}\right)$.

Let us characterize the associated space $V_{h}$ defined by (3.4). Clearly a function $v_{h} \in X_{h}$ belongs to the space $V_{h}$ iff:

(i) for any pair $\left(K_{1}, K_{2}\right)$ of adjacent triangles of $T_{h}$, we have

$$
\forall \mu \in P_{k-1}, \quad \int_{K^{\prime}} \mu\left(v_{h, 1}-v_{h, 2}\right) d \gamma=0,
$$

where $K^{\prime}=K_{1} \cap K_{2}$ and $v_{h, i}$ is the restriction of $v_{h}$ to $K_{i}, i=1,2$;

(ii) for any side $K^{\prime}$ of $T_{h}$ contained in $\Gamma$, we have 


$$
\forall \mu \in P_{k-1}, \quad \int_{K^{\prime}} \mu v_{h} d \gamma=0
$$

Now, in each above integral, $\mu v_{h \mid K^{\prime}}$ belongs to the space $P_{2 k-1 \mid K}$, so that $\int_{K^{\prime}} \mu v_{h} d \gamma$ can be computed exactly in terms of the values of $\mu v_{h}$ at the $k$ Gauss-Legendre quadrature points of the side $K^{\prime}$. Hence a function $v_{h} \in X_{h}$ belongs to $V_{h}$ iff:

$v_{h}$ is continuous at these Gauss-Legendre points contained in $\Omega$;

$v_{h}$ vanishes at these Gauss-Legendre points located on $\Gamma$.

Denote by $\left\{b_{i}\right\}_{i=1}^{k}$ (resp. $\left\{b_{i}\right\}_{i=k+1}^{2 k},\left\{b_{i}\right\}_{i=2 k+1}^{3 k}$ ) the set of the $k$ Gauss-Legendre points of the side $\left[a_{1}, a_{2}\right]$ (resp. $\left[a_{2}, a_{3}\right],\left[a_{3}, a_{1}\right]$ ).

Lemma 5. Assume that $k$ is an odd integer. Then $\left\{b_{i}\right\}_{i=1}^{3 k}$ is $a \hat{T}_{k}$-unisolvent set. Moreover, for $k \geqslant 3$ and for any $P_{k-3}$-unisolvent set $\left\{b_{i}\right\}_{i=3 k+1}^{N(k)}$ of points of $\hat{K}$ $(N(k)=(k+1)(k+2) / 2)$, the set

$$
\hat{\Sigma}=\left\{b_{i}\right\}_{i=1}^{N(k)} \text { is } P_{k} \text {-unisolvent. } *
$$

Proof. Let us first show that $\left\{b_{i}\right\}_{i=1}^{3 k}$ is a $\hat{T}_{k}$-unisolvent set. Since $\operatorname{dim}\left(\hat{T}_{k}\right)=$ $3 k$, we have only to show that a function $v \in \hat{T}_{k}$ which vanishes at the points $b_{i}$, $1 \leqslant i \leqslant 3 k$, must vanish identically. In fact, since $k$ is odd, we get for such a function $v$

$$
v\left(a_{1}\right)=-v\left(a_{2}\right)=v\left(a_{3}\right)=-v\left(a_{1}\right)
$$

so that $v\left(a_{i}\right)=0,1 \leqslant i \leqslant 3$. Hence, the restriction of $v$ to any side $\hat{K}^{\prime}$ of $\hat{K}$ is a polynomial of degree $\leqslant k$ which vanishes at $k+2$ distinct points so that $v=0$.

Next, assume $k \geqslant 3$; we get

$$
N(k)=\operatorname{dim}\left(P_{k}\right)=3 k+\operatorname{dim}\left(P_{k-3}\right) .
$$

Let $\left\{b_{i}\right\}_{i=3 k+1}^{N(k)}$ be a set of $\operatorname{dim}\left(P_{k-3}\right)$ distinct points of $\stackrel{\circ}{\hat{K}}$. Then, given a function $v \in P_{k}$ which vanishes on $\hat{\Sigma}=\left\{b_{i}\right\}_{i=1}^{N(k)}$, we get by the first part of the proof $v_{\mid \partial \hat{K}}=$ 0 so that

$$
v=\lambda_{1} \lambda_{2} \lambda_{3} w, \quad w \in P_{k-3}
$$

Therefore $v=0$ iff $w=0$ or, equivalently, the set $\hat{\Sigma}$ is $P_{k}$-unisolvent iff the set $\left\{b_{i}\right\}_{i=3 k+1}^{N(k)}$ is $P_{k-3}$-unisolvent.

Using the terminology of [4], we deduce from conditions (4.9), (4.10) and Lemma 5 that the space $V_{h}$ is associated with a reference finite element $\left(\hat{K}, \hat{\Sigma}, P_{k}\right)$ where $\hat{\Sigma}$ is given (in a nonunique way for $k \geqslant 3$ ) by Lemma 5 . Setting

$$
\Sigma_{K}=F_{K}(\hat{\Sigma}), \quad K \in T_{h}, \quad \Sigma_{h}=\bigcup_{K \in T_{h}} \Sigma_{K},
$$

we see that the degrees of freedom of a function $v_{h} \in V_{h}$ are its values at the points of $\Sigma_{h} \cap \Omega$. Since $\left(\hat{K}, \hat{\Sigma}, P_{k}\right)$ is not a $C^{0}$ element, the space $V_{h}$ is not contained in $H_{0}^{1}(\Omega)$ and problem $\left(\mathrm{P}_{h}\right)$ is indeed a nonconforming method for solving problem (1.1).

* Let us recall that a set $\Sigma=\left\{a_{i}\right\}_{i=1}^{N}$ is $P$-unisolvent if for any set of scalars $\alpha_{i}, 1 \leqslant i \leqslant N$, there exists a unique function $p \in P$ such that $p\left(a_{i}\right)=\alpha_{i}, 1 \leqslant i \leqslant N$. 
On the other hand, we may choose the degrees of freedom of a function $\mu_{h} \in$ $M_{h}$ to be its values at the $k$ Gauss-Legendre points of each side $K^{\prime}$ of $T_{h}$.

The first hybrid elements are described in Figure 1 where we have used the following conventions for the degrees of freedom:

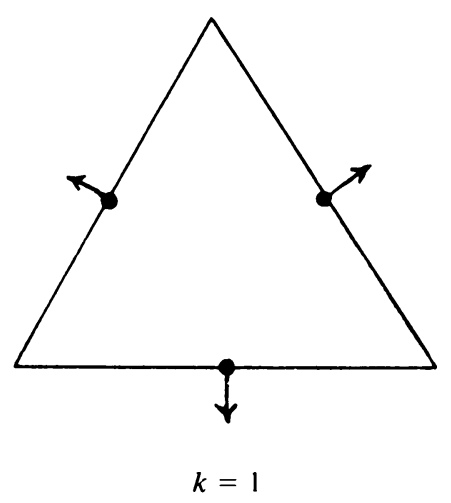

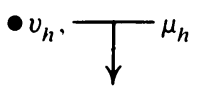

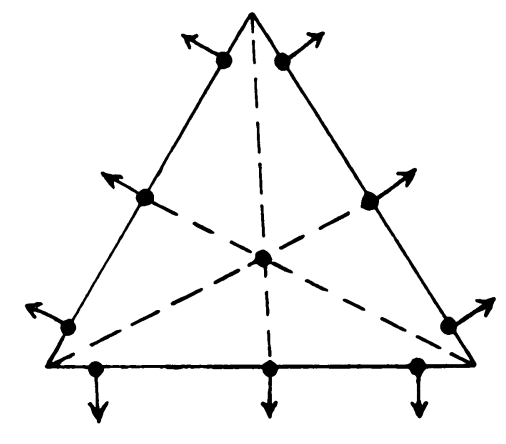

$k=3$

FIGURE 1

These hybrid elements can be considered as nonconforming elements using Loof connections (cf. Crouzeix and Raviart [6], Irons and Razzaque [8]).

Example 2. Let $k \geqslant 2$ be an even integer. By Lemma 4 , the choice $\hat{P}=P_{k}$, $\hat{S}=\hat{S}_{m}$ is suitable only for $k \geqslant m+2$. However, the next result will enable us to construct a hybrid method where

$$
P_{k} \subset \hat{P} \subset P_{k+1}, \quad \hat{S}=\hat{S}_{k-1} .
$$

Lemma 6. Assume that $k$ is an even integer. Define $\hat{P}$ to be the space of polynomials spanned by $P_{k}$ and the function

$$
v_{0}=\left(\lambda_{1}-\lambda_{2}\right)\left(\lambda_{2}-\lambda_{3}\right)\left(\lambda_{3}-\lambda_{1}\right)\left\{\left(\lambda_{1} \lambda_{2}\right)^{(k-2) / 2}\right.
$$

Then, the pair of spaces $\left(\hat{P}, \hat{S}_{k-1}\right)$ satisfies condition (3.15).

Proof. Let $\mu$ be a function of $\hat{S}_{k-1}$ such that

$$
\forall v \in \hat{P}, \quad \int_{\partial \hat{K}} \mu v d \gamma=0 .
$$

Assume again for convenience that the triangle $\hat{K}$ is equilateral. Then, by Remark 3 , $\mu$ is necessarily of the form (4.8). Hence, it is sufficient to prove that

$$
\sum_{i=1}^{3} \int_{\left[a_{i}, a_{i+1}\right]} p_{k-1}\left(\lambda_{i}, \lambda_{i+1}\right) v_{0} d \gamma \neq 0 \quad\left(a_{4}=a_{1}, \lambda_{4}=\lambda_{1}\right) .
$$

Since these three integrals are equal, we have only to check that

$$
\int_{\left[a_{1}, a_{2}\right]} p_{k-1}\left(\lambda_{1}, \lambda_{2}\right) v_{0} d \gamma \neq 0
$$

By an obvious change of variable, the previous integral becomes

$$
\int_{0}^{1} q_{k-1}(\xi) r(\xi) \xi(1-\xi) d \xi,
$$


where

$$
q_{k-1}(\xi)=p_{k-1}(\xi, 1-\xi), \quad r(\xi)=(1-2 \xi)(\xi(1-\xi))^{(k-2) / 2}
$$

Since the roots of $q_{k-1}$ are the Gauss-Lobatto abscissae $\xi_{1}, \ldots, \xi_{k-1}$, the polynomial $q_{k-1}$ is orthogonal to all polynomials of degree $\leqslant k-2$ with respect to the weight function $\xi(1-\xi)$. Now, $r$ is a polynomial of degree $k-1$ so that

$$
\int_{0}^{1} q_{k-1}(\xi) r(\xi) \xi(1-\xi) d \xi \neq 0 .
$$

Otherwise, $q_{k-1}$ would be orthogonal to all polynomials of degree $\leqslant k-1$ which is clearly impossible.

Now, we use the pair of spaces $\left(\hat{P}, \hat{S}_{k-1}\right)$ defined in Lemma 6 in order to construct the spaces $X_{h}$ and $M_{h}$ associated with the triangulation $T_{h}$. Again, by Lemmas 2, 3 and 6, problem $\left(\mathrm{Q}_{h}\right)$ has a unique solution $\left(u_{h}, \lambda_{h}\right)$.

Since, for $v_{h} \in X_{h}, \mu_{h} \in M_{h}$, we have $\mu_{h} v_{h \mid K^{\prime}} \in P_{2 k \mid K^{\prime}}$, the integrals $\int_{K^{\prime}} \mu_{h} v_{h} d \gamma$ cannot be anymore computed exactly in terms of the values of $\mu_{h} v_{h}$ at the $k$ GaussLegendre points of the side $K^{\prime}$. As a consequence, the space $V_{h}$ is not associated here with a (nonconforming) reference finite element $(\hat{K}, \hat{\Sigma}, \hat{P})$ so that the degrees of freedom of a function $v_{h} \in V_{h}$ cannot be determined in a simple way. However, we shall see in Section 7 how the use of numerical quadrature for evaluating the various integrals $\int_{K}{ }^{\prime} \mu_{h} v_{h} d \gamma$ will enable us to solve this difficulty.

5. Examples of Hybrid Quadrilateral Elements. We now consider the case of quadrilateral elements: Here $\hat{K}$ is the unit square $[0,1]^{2}$ in the $(\xi, \eta)$-plane with vertices $a_{1}=(0,0), a_{2}=(1,0), a_{3}=(1,1), a_{4}=(0,1)$. For any integer $k \geqslant 1$, we define $Q_{k}$ to be the space of all polynomials of the form

$$
p(\xi, \eta)=\sum_{0 \leqslant i, j \leqslant k} c_{i j} \xi^{i} \eta^{j}
$$

Lemma 7. Assume that $\hat{K}$ is the unit square. Let $k \geqslant 1$ be an integer. Then the space of functions $\mu \in \hat{S}_{k-1}$ such that

$$
\forall v \in \hat{T}_{k}, \quad \int_{\partial} \mu v d \gamma=0
$$

is one-dimensional.

The proof goes along the same lines of that of Lemma 4. More precisely, using the notations of Section 4 , one can show that the functions $\mu \in \hat{S}_{k-1}$ which satisfy condition (5.1) are given by

$$
\left\{\begin{array}{l}
\mu_{12}=c_{12} p_{k-1}(\xi, 1-\xi), \\
\mu_{23}=c_{23} p_{k-1}(\eta, 1-\eta), \\
\mu_{34}=c_{34} p_{k-1}(1-\xi, \xi), \\
\mu_{41}=c_{41} p_{k-1}(1-\eta, \eta),
\end{array}\right.
$$

with 


$$
\begin{cases}c_{12}=-c_{23}=c_{34}=-c_{41}=c & \text { when } k \text { is odd } \\ c_{12}=c_{23}=c_{34}=c_{41}=c & \text { when } k \text { is even } .\end{cases}
$$

Here again, $\mu_{i j}$ denotes the restriction of $\mu$ to the side $\left[a_{i}, a_{j}\right]$ of $\hat{K}$.

Now, in order to construct a suitable hybrid element, we introduce a space $\hat{Q}$ of functions defined over $\hat{K}$ such that

$$
P_{k} \subset \hat{Q}, \quad \hat{T}_{k} \subset \hat{Q}_{1 \partial \hat{K}} .
$$

Since $\hat{T}_{k}=Q_{k \mid \partial \hat{K}}$, we can choose $\hat{Q}=Q_{k}$. More generally, we can choose "serendipity" space $\hat{Q}$ with $P_{k} \subset \hat{Q} \subset Q_{k}$ : for the derivation of the "serendipity" spaces, we refer to [4] and [20, Chapter 7].

Lemma 8. Let $k \geqslant 1$ be an integer. Define $\hat{P}$ to be the space of polynomials spanned by $\hat{Q}$ and the function

$$
(5.5) v_{0}(\xi, \eta)= \begin{cases}{[\xi(1-\xi)-\eta(1-\eta)]\left[(\xi(1-\xi))^{(k-1) / 2}+(\eta(1-\eta))^{(k-1) / 2}\right]} & \text { when } k \text { is odd, } \\ {[\xi(1-\xi)-\eta(1-\eta)](2 \xi-1)(2 \eta-1)} & \\ \cdot\left[(\xi(1-\xi))^{(k-2) / 2}+(\eta(1-\eta))^{(k-2) / 2}\right] & \text { when } k \text { is even. }\end{cases}
$$

Assume that condition (5.4) holds. Then the pair of spaces $\left(\hat{P}, \hat{S}_{k+1}\right)$ satisfies the compatibility condition (3.16).

The proof is based upon Lemma 7 and is very similar to that of Lemma 6 .

Example 3. Let $k \geqslant 1$ be any integer. Assume that $T_{h}$ is a triangulation of $\bar{\Omega}$ made up with parallelograms $K$. On the reference unit square $\hat{K}$, we choose a pair of spaces $\left(\hat{P}, \hat{S}_{k-1}\right)$ defined in Lemma 8 in order to construct the spaces $X_{h}$ and $M_{h}$. By Lemmas 2, 3 and 8 , problem $\left(Q_{h}\right)$ has a unique solution.

Note that, as in Example 2, the space $V_{h}$ is not associated with a (nonconforming) reference finite element $(\hat{K}, \hat{\Sigma}, \hat{P})$ but see again Section 7 for the use of numerical quadrature.

6. Error Estimates. Let us go back to the general situation of Section 3. We want to derive bounds for the errors $u-u_{h}$ and $\lambda-\lambda_{h}$. We begin by defining more convenient norms over the spaces $X$ and $M$. We first provide $X$ with the norm

$$
\|v\|_{X}=\left(\sum_{K \in T_{h}}\|v\|_{1, K}^{2}\right)^{1 / 2}
$$

where for any $K \in T_{h}$

$$
\begin{gathered}
\|v\|_{1, K}=\left(|v|_{1, K}^{2}+h_{K}^{-2}\|v\|_{0, K}^{2}\right)^{1 / 2}, \\
h_{K}=\text { diameter of } K .
\end{gathered}
$$

Next, we introduce the following norm over the space $M$ 


$$
\|\mu\|_{M}=\sup _{v \in X} \frac{b(v, \mu)}{\|v\|_{X}}
$$

Remark 4. One can easily check that the natural norm (2.7) over $M$ can be equivalently defined by

$$
\|\mu\|_{M}=\sup _{v \in X} \frac{b(v, \mu)}{\|v\|_{X}}
$$

Therefore, the two norms (2.7) and (6.4) are equivalent, the latter being more appropriate for deriving practical error bounds.

Let us now state the following result which can be viewed as a variant of a general theorem of Brezzi concerning the approximation of variational problems (cf. [1, Theorem 2.1]).

Theorem 3. Assume that the hypotheses (3.1) and (3.6) hold. Then, the solution $u_{h} \in V_{h}$ of problem $\left(\mathrm{P}_{h}\right)$ satisfies

$$
\left\|u-u_{h}\right\|_{h}=\left\{\left(\inf _{v_{h} \in V_{h}}\left\|u-v_{h}\right\|_{h}\right)^{2}+\left(\inf _{\mu_{h} \in M_{h}} \sup _{\mu_{h} \in M_{h}} \frac{b\left(v_{h}, \lambda-\mu_{h}\right)}{\left\|v_{h}\right\|_{h}}\right)^{2}\right\}^{1 / 2}
$$

Assume, in addition, that there exists a constant $\alpha>0$ such that

$$
\forall \mu_{h} \in M_{h}, \quad \sup _{v_{h} \in X_{h}} \frac{b\left(v_{h}, \mu_{h}\right)}{\left\|v_{h}\right\|_{X}} \geqslant \alpha\left\|\mu_{h}\right\|_{M} .
$$

Then, problem $\left(\mathrm{Q}_{h}\right)$ has a unique solution $\left(u_{h}, \lambda_{h}\right)$ and we have

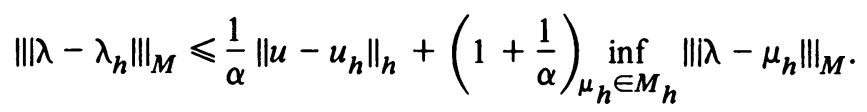

Proof. Assume that hypotheses (3.1) and (3.6) hold so that, by Theorem 2, problem $\left(\mathrm{P}_{h}\right)$ has a unique solution $u_{h} \in V_{h}$. Define $\pi_{h} u \in V_{h}$ by

$$
\forall v_{h} \in V_{h}, \quad a\left(u-\pi_{h} u, v_{h}\right)=0 .
$$

Thus, we may write

$$
\left\|u-u_{h}\right\|_{h}^{2}=\left\|u-\pi_{h} u\right\|_{h}^{2}+\left\|\pi_{h} u-u\right\|_{h}^{2} .
$$

On the other hand, since $\pi_{h} u$ is the orthogonal projection of $u$ upon $V_{h}$ with respect to the inner product $a(\cdot, \cdot)$, we get

$$
\left\|u-\pi_{h} u\right\|_{h}=\inf _{v_{h} \in V_{h}}\left\|u-v_{h}\right\|_{h} .
$$

On the other hand, we have

$$
\left\|\pi_{h} u-u_{h}\right\|_{h}=\sup _{v_{h} \in V_{h}} \frac{a\left(\pi_{h} u-u_{h}, v_{h}\right)}{\left\|v_{h}\right\|_{h}}=\sup _{v_{h} \in V_{h}} \frac{a\left(u-u_{h}, v_{h}\right)}{\left\|v_{h}\right\|_{h}} .
$$


Using (2.14), (3.4) and (3.5), we obtain for all $v_{h} \in V_{h}$ and all $\mu_{h} \in M_{h}$

$$
a\left(u-u_{h}, v_{h}\right)=b\left(v_{h}, \lambda-\mu_{h}\right)
$$

so that

$$
\left\|\pi_{h} u-u_{h}\right\|_{h}=\inf _{\mu_{h} \in M_{h}} \sup _{v_{h} \in V_{h}} \frac{b\left(v_{h}, \lambda-\mu_{h}\right)}{\left\|v_{h}\right\|_{h}} .
$$

This proves (6.6).

Next, we notice that condition (3.7) exactly means that there exists a constant $\alpha>0$ (which may depend on $X_{h}$ and $M_{h}$ ) such that (6.7) holds. Hence, by Theorem 2 again, the hypothesis (6.7) implies the existence and uniqueness of the solution $\left(u_{h}, \lambda_{h}\right)$ of problem $\left(Q_{h}\right)$. Now, using (2.14) and (3.2), we get for all $v_{h} \in X_{h}$ and all $\mu_{h} \in M_{h}$

$$
b\left(v_{h}, \lambda_{h}-\mu_{h}\right)=a\left(u-u_{h}, v_{h}\right)+b\left(v_{h}, \lambda-\mu_{h}\right)
$$

Given $\mu_{h} \in M_{h}$, we can choose by hypothesis (6.7) a function $v_{h} \in X_{h}$ such that

$$
b\left(v_{h}, \lambda_{h}-\mu_{h}\right) \geqslant \alpha\left\|v_{h}\right\|_{X}\left\|\lambda_{h}-\mu_{h}\right\|_{M}
$$

Since $\left\|v_{h}\right\|_{h} \leqslant\left\|v_{h}\right\|_{X}$, we obtain

$$
\alpha\left\|\lambda_{h}-\mu_{h}\right\|_{M} \leqslant\left\|u-u_{h}\right\|_{h}+\left\|\lambda-\mu_{h}\right\|_{M} .
$$

Thus, we get, for all $\mu_{h} \in M_{h}$,

$$
\left\|\lambda \lambda-\lambda_{h}\right\|_{M} \leqslant \frac{1}{\alpha}\left\|u-u_{h}\right\|_{h}+\left(1+\frac{1}{\alpha}\right)\left\|\lambda-\mu_{h}\right\|_{M}
$$

and the desired inequality (6.8) holds.

To apply the above results, we consider a regular family $\left(T_{h}\right)$ of triangulations of the domain $\bar{\Omega}$ in the sense of [5], in that there exists a constant $\sigma>0$ independent of $h$ such that

$$
\max _{K \in T_{h}} \frac{h_{K}}{\rho_{K}} \leqslant \sigma
$$

where

$$
\rho_{K}=\text { diameter of the inscribed sphere in } K \text {. }
$$

Given a function $\varphi \in H^{2}(\Omega)$, we define $\psi \in M$ by

$$
\psi=\frac{\partial \varphi}{\partial v_{K}} \quad \text { on } \partial K, K \in T_{h}
$$

We first evaluate the expression

$$
\inf _{\mu_{h} \in M_{h}} \sup _{v \in X} \frac{b\left(v, \psi-\mu_{h}\right)}{\|v\|_{h}} .
$$

Lemma 9. Let there be given spaces $M_{h}$ defined as in (3.15) which are associated with a regular family of triangulations $\left(T_{h}\right)$. Assume that the inclusion (3.12) 
holds and that $\varphi \in H^{l+1}(\Omega)$ with $1 \leqslant l \leqslant m+1$. Then there exists a constant $T>0$ independent of $h$ such that

$$
\inf _{\mu_{h} \in M_{h}} \sup _{v \in X} \frac{b\left(v, \psi-\mu_{h}\right)}{\|v\|_{h}} \leqslant T h^{l}|\varphi|_{l+1, \Omega}
$$

Proof. Let us recall the following result [6, Lemma 3]: there exists a constant $c>0$ such that, for any $K \in T_{h}$ and for any $(n-1)$-dimensional face $K^{\prime}$, we have $\forall \chi \in H^{l}(K), 1 \leqslant l \leqslant m+1, \forall v \in H^{l}(K), \int_{K^{\prime}}\left(\chi-\pi_{K^{\prime}}^{m} \chi\right) v d \gamma \leqslant c \frac{h_{K}^{l+1}}{\rho_{K}}|\chi|_{1, K}|v|_{1, K}$ where $\pi_{K^{\prime}}^{m}$ denotes the orthogonal projector in $L^{2}\left(K^{\prime}\right)$ upon $P_{\left.m\right|_{K}}{ }^{\prime}$

Now, given $\varphi \in H^{l+1}(\Omega)$, we define $\mu_{h} \in M_{h}$ in the following way: for any $K \in$ $T_{h}$ and any $(n-1)$-dimensional face $K^{\prime}$ of $K$, we set

$$
\mu_{h}=\pi_{K^{\prime}}^{m} \frac{\partial \varphi}{\partial \nu_{K}}=\pi_{K^{\prime}}^{m} \psi \quad \text { on } K^{\prime}
$$

By the previous inequality, we obtain for all $v \in H^{1}(K)$

$$
\int_{K^{\prime}}\left(\psi-\mu_{h}\right) v d \gamma=\int_{K^{\prime}}\left(\frac{\partial \varphi}{\partial \nu_{K}}-\pi_{K^{\prime}}^{m} \frac{\partial \varphi}{\partial \nu_{K}}\right) v d \gamma \leqslant c \frac{h_{K}^{l+1}}{\rho_{K}}|\varphi|_{l+1, K}|v|_{1, K} .
$$

Hence, using (6.9), we get, for all $v \in X$,

$$
b\left(v, \psi-\mu_{h}\right)=-\sum_{K \in T_{h}} \int_{\partial K}\left(\psi-\mu_{h}\right) v d \gamma \leqslant T h^{l}|\varphi|_{l+1, \Omega}\|v\|_{h}
$$

and the conclusion follows immediately. $\square$

We now give an estimate of the error $\left\|u-u_{h}\right\|_{h}$.

THEOREM 4. Let there be given spaces $X_{h}$ and $M_{h}$ defined as in (3.11) and (3.15), which are associated with a regular family of triangulations. Assume that the inclusions (3.8), (3.9), (3.12) hold and that $u \in H^{l+1}(\Omega) \cap H_{0}^{1}(\Omega)$ with $l+1-n / 2$ $>0$ and $1 \leqslant l \leqslant \min (k, m+1)$. Then there exists a constant $T>0$ independent of $h$ such that

$$
\left\|u-u_{h}\right\|_{h} \leqslant T h^{l}|u|_{l+1, \Omega}
$$

Proof. Define the subspace $W_{h}$ of the space $H_{0}^{1}(\Omega)$ by

$$
w_{h}=\left\{w_{h} \in C^{0}(\bar{\Omega}) ; \forall K \in T_{h}, w_{h \mid K} \in P_{K}, w_{h \mid \Gamma}=0\right\} \text {. }
$$

Since $M_{h} \subset M$, we have $W_{h} \subset V_{h}$. Now, in view of the inclusions (3.8), (3.9) and since $\left(T_{h}\right)$ is a regular family, it is well known (see [5, Theorem 5], [4], for example) that, for $u \in H^{l+1}(\Omega)_{n} \cap H_{0}^{l}(\Omega)$ with $l+1-n / 2>0$ and $1 \leqslant l \leqslant k$, we have

$$
\inf _{w_{h} \in W_{h}}\left|u-w_{h}\right|_{1, \Omega} \leqslant c_{1} h^{l}|u|_{l+1, \Omega}
$$

where the constant $c_{1}$ is independent of $h$. Hence 


$$
\inf _{v_{h} \in V_{h}}\left\|u-v_{h}\right\|_{h} \leqslant \inf _{w_{h} \in W_{h}}\left|u-v_{h}\right|_{1, \Omega} \leqslant c_{1} h^{l}|u|_{l+1, \Omega} .
$$

Using next Lemma 9 with $\varphi=u, \psi=\lambda$ and since $u \in H^{l+1}(\Omega)$ with $1 \leqslant l \leqslant m+1$, we obtain for some constant $c_{2}$ independent of $h$

$$
\inf _{\mu_{h} \in M_{h}} \sup _{v_{h} \in V_{h}} \frac{b\left(v_{h}, \lambda-\mu_{h}\right)}{\left\|v_{h}\right\|_{h}} \leqslant c_{2} h^{l}|u|_{l+1, \Omega} .
$$

Then inequality (6.13) follows from inequalities (6.6), (6.14) and (6.15).

Remark 5. Assume in addition that the polyhedral domain $\bar{\Omega}$ is convex. Then, as in [6, Theorem 4], an extension of the classical duality technique of Aubin-Nitsche yields the following $L^{2}$-estimate

$$
\left\|u-u_{h}\right\|_{0, \Omega} \leqslant T^{\prime} h^{l+1}|u|_{l+1, \Omega}
$$

for another constant $T^{\prime}$ independent of $h$. In the case of a general polyhedral domain, the weaker estimate

$$
\left\|u-u_{h}\right\|_{0, \Omega} \leqslant T^{\prime} h^{l}|u|_{l+1, \Omega}
$$

follows from (6.13) and the analogue of the Poincare-Friedrichs inequality

$$
\forall v_{h} \in V_{h}, \quad\left\|v_{h}\right\|_{0, \Omega} \leqslant c\left\|v_{h}\right\|_{h},
$$

where the constant $c=c(\Omega)$ depends only on $\Omega$.

In order to estimate the error $\lambda-\lambda_{h}$, we need first to check that hypothesis (6.7) holds with a constant $\alpha>0$ independent of $h$.

Lemma 10. Let there be given spaces $X_{h}$ and $M_{h}$ defined as in (3.11) and (3.15), which are associated with a regular family of triangulations. Assume that conditions (3.13) and (3.16) hold. Then hypothesis (6.7) holds with a constant $\alpha>0$ independent of $h$.

Proof. For all $K \in T_{h}$ and all $\mu \in H^{-1 / 2}(\partial K)$, we define

$$
\|\mu\|_{\partial K}=\sup _{w \in H^{l}(K)} \frac{\int_{\partial K} \mu w d \gamma}{\|w\|_{1, K}} .
$$

Then condition (3.16) means that there exists a constant $\hat{\alpha}>0$ such that

$$
\forall \hat{\mu} \in \hat{S}, \quad \sup _{\hat{w} \in \hat{P}} \frac{\int_{\partial \hat{K}} \hat{\mu} \hat{w} d \gamma}{\|\hat{w}\|_{1, \hat{K}}} \geqslant \hat{\alpha}\|\hat{\mu}\|_{\partial \hat{K}} .
$$

Let $\mu_{h} \in M_{h}$. The first part of the proof consists in showing that, for each $K \in T_{h}$, there exists a function $v \in P_{K}$ such that

$$
\int_{\partial K} \mu_{h} v d \gamma \geqslant \hat{\alpha} \frac{\rho_{\hat{K}}}{h_{\hat{K}}} \frac{\rho_{K}}{h_{K}}\left\|\mu_{h}\right\|_{\partial K}\|v\|_{1, K} .
$$

Consider the affine mapping $F_{K}: x \rightarrow F_{K}(x)=B_{K} x+b_{K}$, where $B_{K}$ is an invertible element of $L\left(R^{n}\right)$ and $b_{K}$ is a vector in $R^{n}$, such that $K=F_{K}(\hat{K})$; we may write 


$$
\int_{\partial K} \mu_{h} v d \gamma=\int_{\partial \hat{K}} \hat{\lambda} \hat{v} d \gamma
$$

where $\hat{v}=v_{0} F_{K} \in \hat{P}$ and, by (3.13), $\hat{\lambda} \in \hat{S}$. Thus, we choose $\hat{v} \in \hat{P}$ such that

$$
\int_{\partial \hat{K}} \hat{\lambda} \hat{v} d \gamma \geqslant \hat{\alpha}\|\hat{\lambda}\|_{\partial} \hat{K}\|\hat{v}\|_{1, \hat{K}} .
$$

Now, by using [5, Eq. (4.16) and Lemma 2], we obtain

$$
\begin{aligned}
\|v\|_{1, K}^{2} & =|v|_{1, K}^{2}+h_{K}^{-2}\|v\|_{0, K}^{2} \leqslant\left|\operatorname{det}\left(B_{K}\right)\right|\left(\left\|B_{K}^{-1}\right\|^{2}|\hat{v}|_{1, \hat{K}}^{2}+h_{K}^{-2}\|\hat{v}\|_{0, \hat{K}}^{2}\right) \\
& \leqslant\left|\operatorname{det}\left(B_{K}\right)\right|\left(h_{\hat{R}}^{2} \rho_{K}^{-2}|\hat{v}|_{1, \hat{K}}^{2}+h_{K}^{-2}\|\hat{v}\|_{0, \hat{K}}^{2}\right)
\end{aligned}
$$

so that

$$
\|v\|_{1, K} \leqslant h_{\hat{K}} \rho_{K}^{-1}\left|\operatorname{det}\left(B_{K}\right)\right|^{1 / 2}\|\hat{i v}\|_{1, \hat{K}} .
$$

On the other hand, it follows from (6.19) and (6.22) that

$$
\left\|\mu_{h}\right\|_{\partial K}=\sup _{w \in H^{l}(K)} \frac{\int_{\partial \hat{K}} \hat{\lambda} \hat{w} d \gamma}{\|w\|_{1, K}}, \quad \hat{w}=w_{0} F_{K} .
$$

Since by [4, Eq. (4.15) and Lemma 2]

$$
\|\hat{w}\|_{1, \hat{K}} \leqslant h_{K} \rho_{\hat{K}}^{-1}\left|\operatorname{det}\left(B_{K}\right)\right|^{-1 / 2}\|w\|_{1, K},
$$

we get

$$
\left\|\mu_{h}\right\|_{\partial K} \leqslant h_{K} \rho_{\hat{K}}^{-1}\left|\operatorname{det}\left(B_{K}\right)\right|^{-1 / 2}\|\hat{\lambda}\|_{\partial \hat{K}} .
$$

Hence inequality (6.21) follows from (6.22)-(6.25).

We shall now show that condition (6.7) follows from (6.21) with

$$
\alpha=\frac{\hat{\alpha}}{\sigma} \frac{\rho_{\hat{K}}}{h_{\hat{K}}} .
$$

Let $w \in H^{l}(K)$; by normalizing $v$ in (6.21), there exists a function $v \in P_{K}$ such that

$$
-\int_{\partial K} \mu_{h} v d \gamma \geqslant-\alpha \int_{\partial K} \mu_{h} w d \gamma, \quad\|v\|_{1, K}=\|w\|_{1, K} .
$$

Therefore, given a function $w \in X$, there exists a function $v_{h} \in X_{h}$ such that

$$
\frac{b\left(v_{h}, \mu_{h}\right)}{\left\|v_{h}\right\|_{X}} \geqslant \alpha \frac{b\left(w, \mu_{h}\right)}{\|w\|_{X}} .
$$

Hence, we have

$$
\sup _{v_{h} \in X_{h}} \frac{b\left(v_{h}, \mu_{h}\right)}{\left\|v_{h}\right\|_{X}} \geqslant \alpha \sup _{w \in X} \frac{b\left(w, \mu_{h}\right)}{\|w\|_{X}}=\alpha\left\|\mu_{h}\right\|_{M} \text {. }
$$


We are now able to prove

TheOREM 5. Assume the hypotheses of Theorem 4. Assume, in addition, that conditions (3.13) and (3.16) hold. Then there exists a constant $T>0$ independent of $h$ such that

$$
\left\|\left.\left|\lambda-\lambda_{h} \|_{M} \leqslant T h^{l}\right| u\right|_{l+1, \Omega}\right.
$$

Proof. Using Lemma 9 with $\varphi=u$ and $\psi=\lambda$ we obtain

$$
\inf _{\mu_{h} \in M_{h}} \sup _{v \in X} \frac{b\left(v, \lambda-\mu_{h}\right)}{\|v\|_{h}} \leqslant T h^{l}|u|_{l+1, \Omega} .
$$

Since $\|v\|_{h} \leqslant\|v\|_{X}$ and by the definition (6.4), we get

$$
\inf _{\mu_{h} \in M_{h}}\left\|i \lambda-\left.\mu_{h}\left|\|_{M} \leqslant T h^{l}\right| u\right|_{l+1, \Omega} .\right.
$$

Then inequality (6.26) follows from Lemma 10 and the inequalities (6.8), (6.13) and (6.27).

Remark 6. In fact, one can derive a more "suggestive" result (cf. [18]). Define the following norm over the space $\Pi_{K \in T_{h}} L^{2}(\partial K)$.

$$
\|\mu\|_{h}=\left(\sum_{K \in T_{h}} h_{K}\|\mu\|_{0, \partial K}^{2}\right)^{1 / 2} .
$$

Then one can prove (cf. [18]) that there exists a constant $c=c(\Omega)>0$ such that

$$
\forall \mu_{h} \in M_{h}, \quad\left\|\mu_{h}\right\|_{h} \leqslant C\left\|\mu_{h}\right\|_{M},
$$

so that we get as a consequence of the inequalities (6.26) and (6.29)

$$
\left\|\lambda-\lambda_{h}\right\|_{h} \leqslant T h^{l}|u|_{l+1, \Omega} \cdot \square
$$

Let us go back to the Examples 1, 2, 3. Assume that $u \in H^{l+1}(\Omega)$ for some integer $l$ with $1 \leqslant l \leqslant k$ and that $\left(T_{h}\right)$ is a regular family of triangulations. It follows from Theorems 4 and 5 that in each example, we have

$$
\left\|u-u_{h}\right\|_{h}+\left\|\left|\lambda-\lambda_{h}\right|\right\|_{M} \leqslant T h^{l}|u|_{l+1, \Omega} .
$$

7. Remarks on Some Nonconforming Methods. Let us go back to the general formulation of primal hybrid methods. It can be of interest to use numerical quadrature for evaluating the various surface integrals $\int_{K^{\prime}} \mu v d \gamma$ which appear in the bilinear form $b(v, \mu)$. Then we obtain a new bilinear form $b_{h}(v, \mu)$ and we replace problem $\left(\mathrm{Q}_{h}\right)$ by the following one, called problem $\left(\mathrm{Q}_{h}^{*}\right)$ : Find a pair $\left(u_{h}^{*}, \lambda_{h}^{*}\right) \in X_{h} \times M_{h}$ such that

$$
\begin{gathered}
\forall v_{h} \in X_{h}, \quad a\left(u_{h}^{*}, v_{h}\right)+b_{h}\left(v_{h}, \lambda_{h}^{*}\right)=\int_{\Omega} f v_{h} d x, \\
\forall \mu_{h} \in M_{h}, \quad b_{h}\left(u_{h}^{*}, \mu_{h}\right)=0 .
\end{gathered}
$$

With the bilinear form $b_{h}(v, \mu)$, we associate the subspace $V_{h}^{*}$ of the space $X_{h}$ 


$$
V_{h}^{*}=\left\{v_{h} \in X_{h} ; \forall \mu_{h} \in M_{h}, b_{h}\left(v_{h}, \mu_{h}\right)=0\right\}
$$

and we define problem $\left(\mathrm{P}_{h}^{*}\right)$ : Find $u_{h}^{*} \in V_{h}^{*}$ such that

$$
\forall v_{h} \in V_{h}^{*}, a\left(u_{h}^{*}, v_{h}\right)=\int_{\Omega} f v_{h} d x .
$$

Here again, if $\left(u_{h}^{*}, \lambda_{h}^{*}\right) \in X_{h} \times M_{h}$ is a solution of problem $\left(\mathrm{Q}_{h}^{*}\right), u_{h}^{*} \in V_{h}^{*}$ is a solution of problem $\left(\mathrm{P}_{h}^{*}\right)$. Now, the degrees of freedom of a function $v_{h} \in V_{h}^{*}$ can be easier to determine than those of a function $v_{h} \in V_{h}$ so that problem $\left(\mathrm{P}_{h}^{*}\right)$ can be simpler to solve than problem $\left(\mathrm{P}_{h}\right)$.

This is indeed the case in some important examples that we shall discuss briefly. Again, we refer to [18] for general results concerning the existence, uniqueness and approximation properties of the solutions of problems $\left(\mathrm{Q}_{h}^{*}\right)$ and $\left(\mathrm{P}_{h}^{*}\right)$.

Example 4. Consider again the situation of Example 2 where $\hat{K}$ is a triangle, $k$ is an even integer and the pair $\left(\hat{P}, \hat{S}_{k-1}\right)$ is chosen as in Lemma 6. But here, for each side $K^{\prime}$ of $T_{h}$, we use the $k$-points Gauss-Legendre quadrature formula to compute the integral $\int_{K} \mu_{h} v_{h} d \gamma, v_{h} \in X_{h}, \mu_{h} \in M_{h}$. In fact, one can prove that the corresponding problem $\left(\mathrm{Q}_{h}^{*}\right)$ has a unique solution $\left(u_{h}^{*}, \lambda_{h}^{*}\right)$ and that, for $u \in H^{k}(\Omega)$, we have the error estimate

$$
\left\|u-u_{h}^{*}\right\|_{h}+\|\| \lambda-\lambda_{h}^{*} \|_{M} \leqslant T h^{k-1}|u|_{k, \Omega} .
$$

Note that the order of accuracy of the hybrid method has been decreased by one.

On the other hand, the space $V_{h}^{*}$ can now be characterized as in Example 1: a function $v_{h} \in X_{h}$ belongs to $V_{h}^{*}$ iff it satisfies conditions (4.9) and (4.10). Moreover, the space $V_{h}^{*}$ is associated with a nonconforming reference finite element $(\hat{K}, \hat{\Sigma}, \hat{P})$. Assume for simplicity that $k=2$. Then:

(i) $\hat{\Sigma}=\left\{b_{i}\right\}_{i=1}^{7}$ where the points $b_{i}, 1 \leqslant i \leqslant 6$, are the Gauss-Legendre quadrature points and where $b_{7}$ may be chosen as the centroid of $\hat{K}$;

(ii) $\hat{P}$ is the space of polynomials spanned by $P_{2}$ and the function $v_{0}=$ $\left(\lambda_{1}-\lambda_{2}\right)\left(\lambda_{2}-\lambda_{3}\right)\left(\lambda_{3}-\lambda_{1}\right)$.

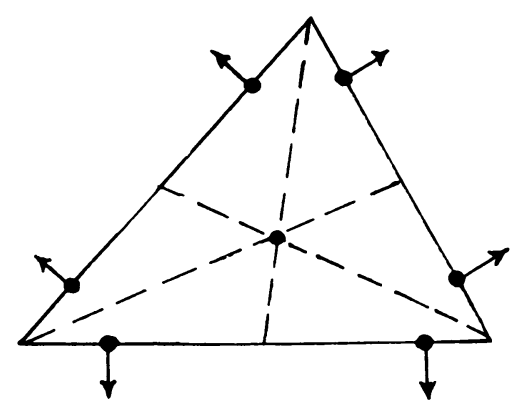

FIGURE 2

We recognize here a nonconforming element introduced by Irons and Razzaque [8, p. 579].

Example 5. We next consider the situation of Example $3: \hat{K}$ is the unit square, $k$ is any integer $\geqslant 1$ and the pair $\left(\hat{P}, \hat{S}_{k-1}\right)$ is chosen as in Lemma 8. Again we compute each integral $\int_{K} \mu_{h} v_{h} d \gamma$ by using the $k$-points Gauss-Legendre quadrature 
formula. Then the problem $\left(\mathrm{Q}_{h}^{*}\right)$ has a unique solution and, for $u \in H^{k}(\Omega)$, we have the estimate (7.5). Note that the convergence of this hybrid method is ensured only for $k \geqslant 2$.

For instance, when $k=2$ and $\hat{Q}$ is the space of polynomials spanned by $1, \xi, \eta$, $\xi^{2}, \xi \eta, \eta^{2}, \xi^{2} \eta, \xi \eta^{2}$, the space $V_{h}^{*}$ is associated with a nonconforming reference element $(\hat{K}, \hat{\Sigma}, \hat{P})$ such that

(i) $\hat{\Sigma}=\left\{b_{i}\right\}_{i=1}^{9}$ where the points $b_{i}, 1 \leqslant i \leqslant 8$, are the Gauss-Legendre quadrature points and where $b_{9}$ is the centroid of $\hat{K}$;

(ii) $\hat{P}$ is the space of polynomials spanned by $\hat{Q}$ and the function $v_{0}(\xi, \eta)=$ $[\xi(1-\xi)-\eta(1-\eta)](2 \xi-1)(2 \eta-1)$.

Here again, we obtain a nonconforming element introduced by Irons and Razzaque [8, p. 579].

Example 6 (Wilson's rectangular element [19]). Here $\hat{K}$ is the unit square; we set $\hat{P}=P_{2}$ and $\hat{S}=\hat{S}_{1}$. Unfortunately, by Lemma 7 , the compatibility condition (3.16) is not satisfied so that the problem $\left(Q_{h}\right)$ is not well posed. However, by Lemma 2, problem $\left(\mathrm{P}_{h}\right)$ has a unique solution $u_{h} \in V_{h}$ and, by using Theorem 4 with $k$ $=1$ and $m=0$, we get the estimate

$$
\left\|u-u_{h}\right\|_{h} \leqslant T h|u|_{2, \Omega} .
$$

Now, instead of computing exactly each integral $\int_{K}{ }^{\prime} \mu_{h} v_{h} d \gamma$, we use the trapezoidal rule, the quadrature nodes being the endpoints of $K^{\prime}$. Then, one can prove that problem $\left(\mathrm{P}_{h}^{*}\right)$ has a unique solution $u_{h}^{*} \in V_{h}^{*}$ and that, for $u \in H^{2}(\Omega)$, we have

$$
\left\|u-u_{h}^{*}\right\|_{h} \leqslant T h|u|_{2, \Omega} .
$$

Furthermore, the space $V_{h}^{*}$ may be characterized as the space of all functions $v_{h}$ such that

(i) for each rectangle $K \in T_{h}, v_{h \mid K} \in P_{2}$;

(ii) $v_{h}$ is continuous at the vertices of $T_{h}$ contained in $\Omega$;

(iii) $v_{h}$ vanishes at the vertices located on $\Gamma$.

Hence the space $V_{h}^{*}$ is associated with Wilson's rectangular element as it is described in [14]. For direct proofs of convergence of this nonconforming method, we refer to $[14],[9]$.

Laboratoire Analyse Numerique L. A. 189

Université P. \& M. Curie (Paris VI)

75230 Paris Cedex 05, France

1. F. BREZZI, "On the existence, uniqueness and approximation of saddle-point problems arising from Lagrangian multipliers," Rev. Française Automat. Informat. Recherche Opérationelle Sér. Anal. Numér., v. 8, 1974, no. R-2, pp. 129-151. MR 51 \#1540.

2. F. BREZZI, "Sur la méthode des éléments finis hybrides pour le problème biharmonique," Numer. Math., v. 24, 1975, pp. 103-151.

3. F. BREZZI \& L. D. MARINI, "On the numerical solution of plate bending problems by hybrid methods," Rev. Française Automat. Informat. Recherche Opérationelle Sér. Anal. Numér., v. 9, 1975 , no. R-3, pp. 5-50.

4. P. G. CIARLET, Numerical Analysis of the Finite Element Method, Séminaire de Mathématiques Supérieures, Univ. de Montréal Press, Montréal, 1975. 
5. P. G. CIARLET \& P. A. RAVIART, "General Lagrange and Hermite interpolation in $R^{n}$ with applications to finite element methods," Arch. Rational Mech. Anal., v. 46, 1972, pp. 177-199. MR 49 \#1730.

6. M. CROUZEIX \& P. A. RAVIART, "Conforming and nonconforming finite element methods for solving the stationary Stokes equations. I," Rev. Française Automat. Informat. Recherche Opérationelle Sér. Anal. Numér., v. 7, 1973, no. R-3, pp. 33-75." MR 49 \#8401.

7. B. FRAEIJS DE VEUBEKE, "Variational principles and the patch test," Internat. J. Numer. Methods Engrg., v. 8, 1974, pp. 783-801. MR 51 \#12099.

8. B. M. IRONS \& A. RAZZAQUE, "Experience with the patch test for convergence of finite elements," The Mathematical Foundations of the Finite Element Method with Applications to Partial Differential Equations, Part II (A. K. Aziz, Editor), Academic Press, New York, 1972, pp. 557-587. MR 49 \#11824.

9. P. LESAINT, “On the convergence of Wilson's nonconforming element for solving the elastic problems," Comput. Methods Appl. Mech. Engrg., v. 7, 1976, pp. 1-16.

10. T. H. H. PIAN, "Formulations of finite element methods for solid continua," Recent Advances in Matrix Methods of Structural Analysis and Design (R. H. Gallagher, Y. Yamada and J. T. Oden, Editors), The University of Alabama Press, 1971, pp. 49-83.

11. T. H. H. PIAN, "Finite element formulation by variational principles with relaxed continuity requirements," The Mathematical Foundations of the Finite Element Method with Applications to Partial Differential Equations, Part II (A. K. Aziz, Editor), Academic Press, New York, 1972, pp. 671-687. MR 49 \#11824.

12. T. H. H. PIAN \& P. TONG, "Basis of finite element methods for solid continua," Internat. J. Numer. Methods. Engrg., v. 1, 1969, pp. 3-28.

13. P. A. RAVIART, "Hybrid finite element methods for solving 2 nd order elliptic equations," Conf. on Numerical Analysis, Royal Irish Academy, Dublin, 1974.

14. G. STRANG, "Variational crimes in the finite element methods," The Mathematical Foundations of the Finite Element Method with Applications to Partial Differential Equations, Part II (A. K. Aziz, Editor), Academic Press, New York, 1972, pp. 689-710. MR 49 \#11824.

15. G. STRANG \& G. J. FIX, An Analysis of the Finite Element Method, Prentice-Hall, Englewood Cliffs, N. J., 1973.

16. J. M. THOMAS, "Méthode des éléments finis hybrides duaux pour les problèmes elliptiques du 2nd ordre," Rev. Française Automat. Informat. Recherche Opérationelle Sér. Anal. Numér., v. 10, 1976, pp. 51-79.

17. J. M. THOMAS, Méthode des Eléments Finis Equilibre pour les Problèmes Elliptiques du 2nd Ordre, Journées des Eléments Finis, May 12-14 1975, Rennes, France.

18. J. M. THOMAS, Thesis, Univ. P. \& M. Curie, Paris, 1977.

19. E. L. WILSON, R. L. TAYLOR, W. P. DOHERTY \& J. GHABOUSSI, "Incompatible displacement models," Sympos. on Numerical Methods in Structural Engineering, O. N. R., University of Illinois, 1971.

20. O. C. ZIENKIEWICZ, The Finite Element Method in Engineering Science, 2nd rev. ed., McGraw-Hill, London, 1971. MR 47 \#418. 